\title{
October 2017 Pulmonary Case of the Month
}

Eric A. Jensen, MD

\author{
Department of Radiology \\ Mayo Clinic Arizona \\ Scottsdale, AZ USA
}

\section{History of Present IIIness}

A 56-year-old woman presented with 3 days of non-productive cough, low-grade fever and severe right-sided pleuritic chest pain.

\section{Past Medical History, Social History and Family History}

She was diagnosed with coccidioidomycosis 5 years previously. She reports that she has had pneumonia every 6 to 12 months since her diagnosis with valley fever. She does not smoke. Family history is noncontributory.

\section{Physical Examination}

Her vital signs were unremarkable and she was afebrile but did cough frequently during the examination. Her lungs were clear and the rest of the physical examination was unremarkable.

\section{Chest Radiography}

She brings in two prior chest x-rays, one from 2011 (Figure 1, Panels A \& B) and another from 2012 (Figure 1, Panel C). 

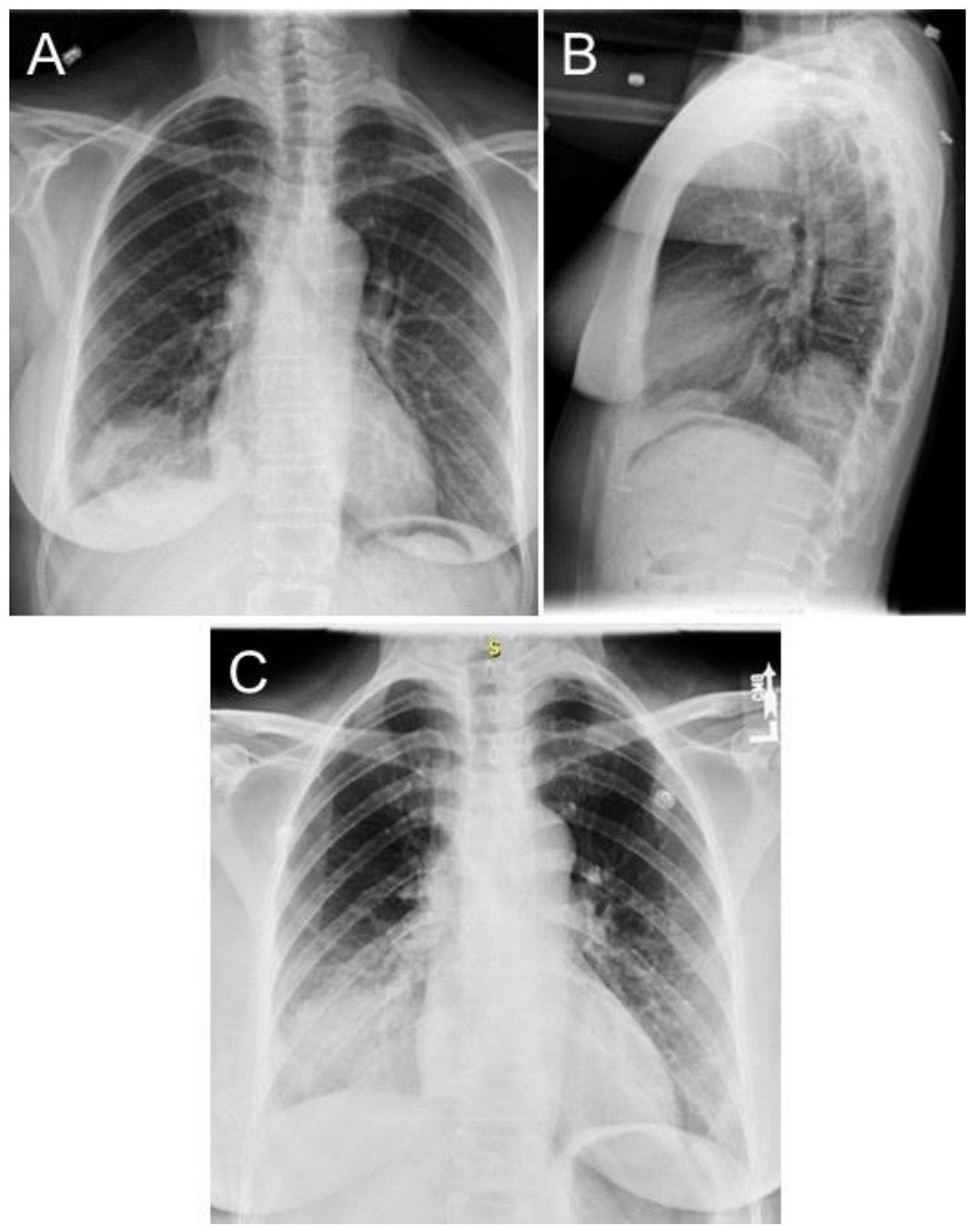

Figure 1. Chest radiograph from 2011 (A \& B) and from 2012 (C).

Which of the following best describes the chest x-rays?

1. A repeat chest $\mathrm{x}$-ray should be performed

2. A right lower lobe mass is present which appears to have enlarged from 2011 to 2012

3. There is a right lower posterior lung density

4. 1 and 3

5. All of the above 


\section{Correct!}

\section{1 and 3}

There is a large right lower lung density which does not appear to have significantly changed from 2011 and 2012. It appears rounded and there are no clear air bronchograms present. A chest x-ray was repeated and was not changed compared to the 2 chest $x$-rays in Figure 1.

What should be done next?

1. Bronchoscopy

2. Needle biopsy of the density

3. Repeat the chest $x$-ray in 6-8 weeks

4. Serologies for coccidioidomycosis

5. Thoracic CT scan 


\section{Correct! \\ 5. Thoracic CT scan}

Of the choices listed, thoracic CT is best. A pneumonia or a lung cancer would certainly have changed in 5 years, either resolving or progressing.

Coccidioidomycosis is also unlikely to cause a density which is unchanged over 5 years and the serologies would likely not be unhelpful. Needle biopsy or bronchoscopy might eventually be needed but not until the density is better defined.

A thoracic CT scan was performed (Figure 2).
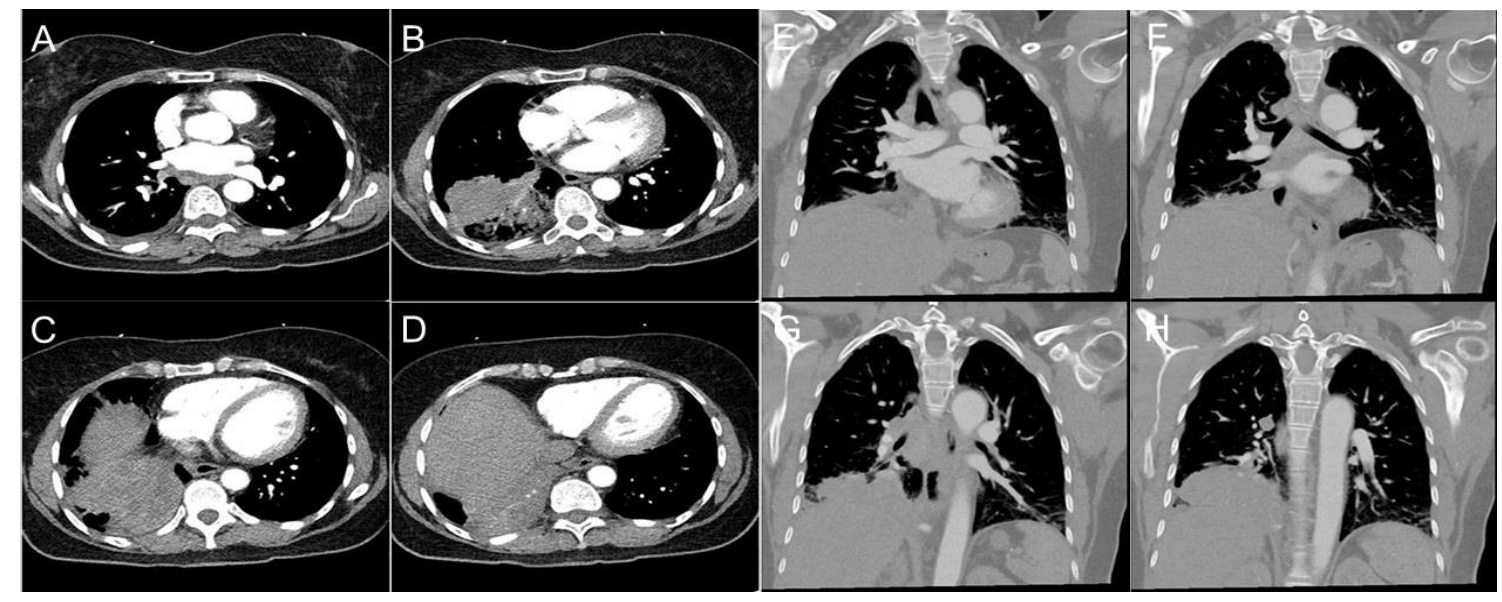

Figure 2. Thoracic CT scans in soft tissue windows. Representative axial (A-D) and coronal $(\mathrm{E}-\mathrm{H})$ images.

Based on the presentation and the thoracic CT findings what is the most likely diagnosis?

1. Bronchiectasis

2. Pulmonary alveolar proteinosis

3. Pulmonary hamartoma

4. Pulmonary sequestration

5. Sarcoidosis 


\section{Correct! \\ 4. Pulmonary sequestration}

On the video images an aberrant pulmonary artery is seen suppling the density. The CT images combined with the history are diagnostic of pulmonary sequestration. Pulmonary sequestration is the aberrant formation of segmental lung tissue that has no connection with the bronchial tree or pulmonary arteries (1). The estimated incidence is $0.1 \%$. There are two types: intralobar sequestration (ILS) and extralobar sequestration (ELS). The abnormal lung tissue is located within the visceral pleura of a pulmonary lobe in ILS, whereas the ELS has its own visceral pleura. ILS accounts for $75-85 \%$ of sequestrations. Sequestration preferentially affects the lower lobes. $60 \%$ of ILS affect the left lower lobe, and $40 \%$ the right lower lobe. ELS almost always affect the left lower lobe, however, about $10 \%$ of can be subdiaphragmatic.

ILS is closely connected to the adjacent normal lung and do not have a separate pleura. In ILS, venous drainage commonly occurs via the pulmonary veins, but can occur through the azygous-hemiazygous system, portal vein, right atrium or inferior vena cava.

ELS is separated from any surrounding lung by its own pleura. Venous drainage most commonly through the systemic veins into the right atrium (but is variable).

Which of the following is $\underline{\text { true }}$ regarding pulmonary sequestration in adults?

1. Is often asymptomatic

2. Can present with right to left shunt physiology

3. Most commonly is ILS rather than ELS

4. 1 and 3

5. All of the above 


\section{Correct! \\ 5. All of the above}

All are true in adult patients. ELS more commonly presents in children and 50 $60 \%$ are associated with congenital pulmonary airway malformation (sometimes termed a hybrid lesion), congenital heart disease or vertebral anomalies, congenital diaphragmatic hernia, or Scimitar syndrome. These associations are rarely true in adult patients.

Traditionally treatment has been surgical resection. ELS with separate pleural investments can usually be removed sparing normal lung tissue. However, with ILS, segmental resection or even lobectomy will be necessary. Embolization has also been successful when a right to left shunt is present. Spontaneous involution has also been reported.

Our patient was offered surgical resection but after hearing the benefits versus the possible complications, she decided to be followed without surgical intervention at this time.

\section{Reference}

1. Abbey P, Das CJ, Pangtey GS, Seith A, Dutta R, Kumar A. Imaging in bronchopulmonary sequestration. J Med Imaging Radiat Oncol. 2009 Feb;53(1):22-31. [CrossRef] [PubMed] 